\title{
Chlorinated Solvents Their Advantages, Disadvantages and Alternatives in Organic and Medicinal Chemistry
}

\author{
Andrew Jordan ${ }^{\mathrm{a}^{*}}$, Patrick Stoy ${ }^{\mathrm{b}}$, and Helen F. Sneddon ${ }^{\mathrm{c}^{*}}$ \\ ${ }^{* a}$ GlaxoSmithKline Carbon Neutral Laboratory for Sustainable Chemistry, Jubilee Campus, University of Nottingham, 6 Triumph Rd, \\ Nottingham NG7 2GA. E-mail: andrew.jordan@nottingham.ac.uk. \\ b Drug Design and Selection, Platform and Technology Sciences, GlaxoSmithKline, 1250 South Collegeville Road, Collegeville, PA 19426, \\ United States \\ ${ }^{{ }^{*} \mathrm{c}}$ GSK, Medicines Research Centre, Gunnels Wood Road, Stevenage, Herts., UK, SG1 2NY. E-mail: helen.f.sneddon@gsk.com.
}

\section{Table of Contents}

Chlorinated Solvents Their Advantages, Disadvantages and Alternatives in Organic and Medicinal Chemistry ………………..........................................................................

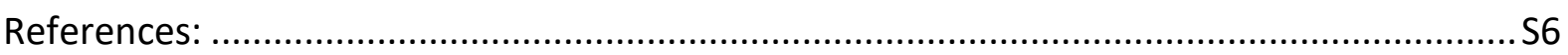

Table S1: Unified Solvent Selection Guide for Reactions of Importance to Medicinal Chemistry 
Table S1: Unified Solvent Selection Guide for Reactions of Importance to Medicinal Chemistry

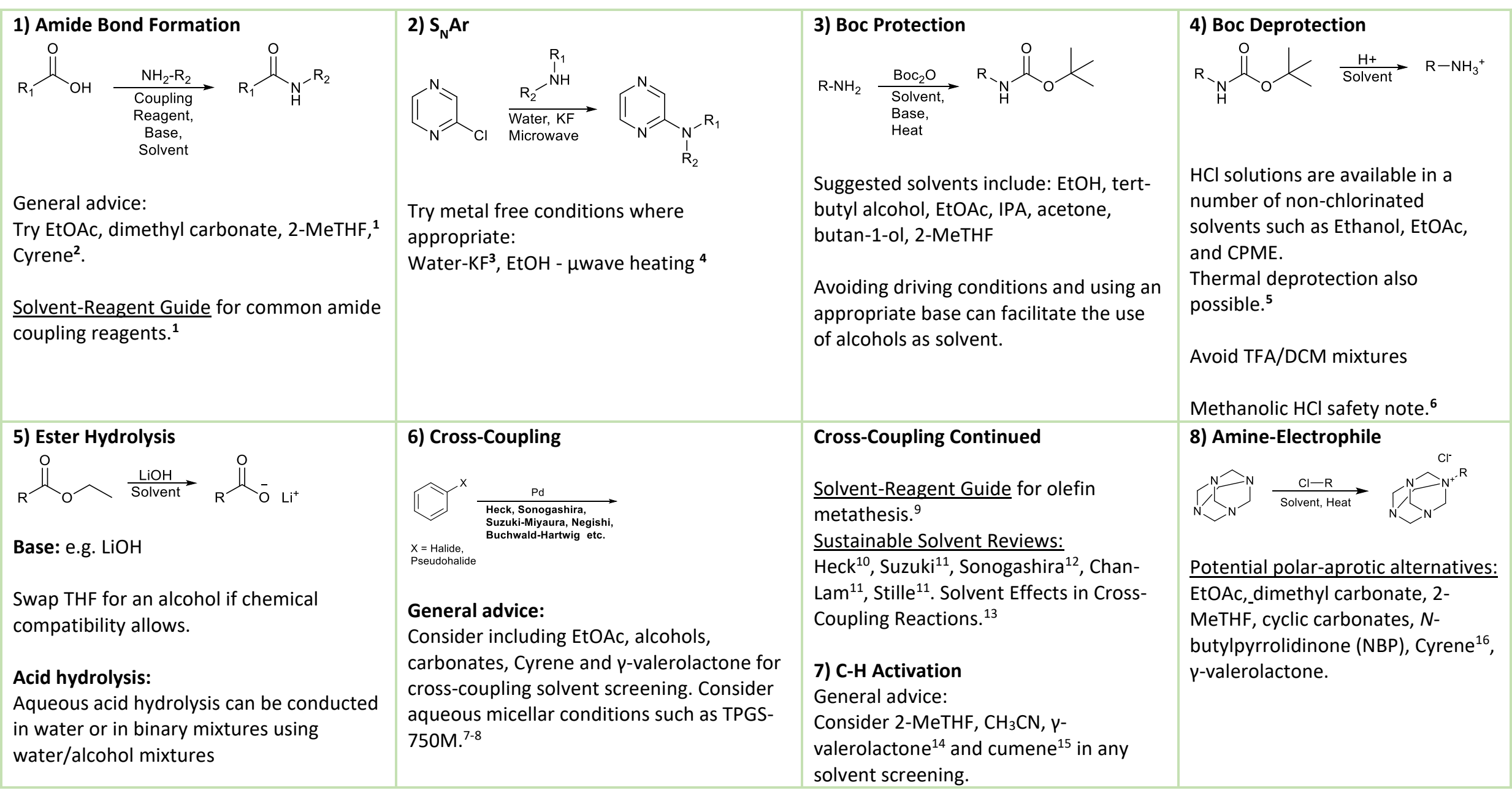




\section{9) Reductive Amination}

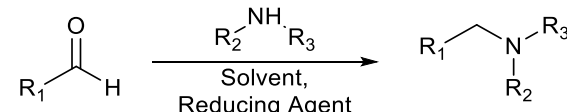

Avoid DCE and try EtOAc as first choice.

Also consider dimethyl carbonate, IPA and 2-MeTHF. ${ }^{17}$

Solvent-Reagent Guide for aldehyde based reductive aminations using boron based reagents. ${ }^{17}$

\section{3) $\mathrm{NO}_{x}$ and Nitrile Reduction}

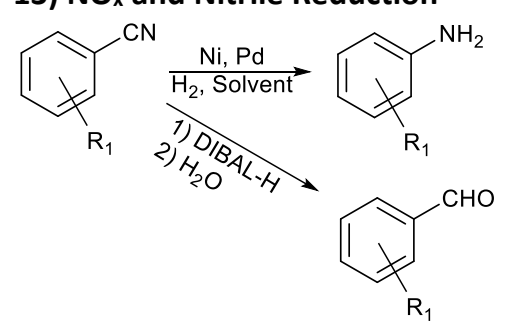

$\mathrm{Pd} / \mathrm{C}+\mathrm{H}_{2}:$ Try EtOH, EtOAc, or water first. ${ }^{21}$ Avoid chlorinated solvents and $\mathrm{MeOH}$ (flammability). For DIBAL-H then heptane and cyclohexane are both preferable to hexane or petroleum ether.<smiles>[R7]c1cccc([N+](=O)[O-])c1CCC(C)OC(C)=O</smiles>

Iron + Acetic Acid: Try solvents such as $\mathrm{EtOH}^{21}$ or EtOAc ${ }^{22}$.

\section{0) Debenzylation}

O<smiles>[R]C([R])=COCc1ccccc1</smiles>

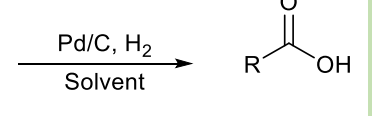

$\mathrm{R}_{1}{ }_{[\mathrm{N}, \widehat{\mathrm{O}]}}$

$\mathrm{Pd} / \mathrm{C}, \mathrm{H}_{2}$

$\mathrm{R}_{1}-\mathrm{OH}$

Or $\mathrm{R}_{1}-\mathrm{NH}_{2}$

Hydrogenolysis $\mathrm{Pd} / \mathrm{C}+\mathrm{H}_{2}$ : Try EtOH, EtOAc, or water first. Avoid chlorinated solvents and $\mathrm{MeOH}$ (flammability).

\section{4) Diazotization}

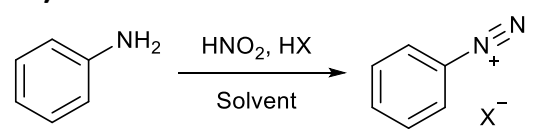

Diazonium $\mathrm{BF}_{4}$ salts can be formed in EtOH. ${ }^{23}$

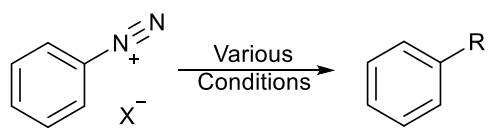

$\mathrm{R}=$ Halides $\mathrm{CF}_{3}, \mathrm{Cyano}, \mathrm{OH}$,

$\mathrm{NH}_{2}, \mathrm{H}, \mathrm{C}$-C Cross-Coupled etc.

Transformations using diazonium salts

often employ polar solvents such as water and $\mathrm{MeOH} .{ }^{24}$ Heck-Matsuda ${ }^{25-26}$ and Suzuki-

Miyaura ${ }^{27-28}$ reactions using diazonium salts have been reported in $\mathrm{EtOH}$ and $\mathrm{MeOH}$.

\section{1) Heteroatom Alkylation}

$\stackrel{\mathrm{R}_{2}-\mathrm{X}}{\text { Base, Solvent }}$

$\mathrm{R}_{1}$

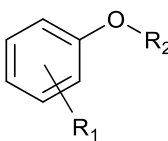

Potential polar-aprotic alternatives:

$\mathrm{N}$-butylpyrrolidinone (NBP), cyclic carbonates, Cyrene ${ }^{16}, \gamma$-valerolactone ${ }^{12}$ Dimethyl carbonate, EtOAc, 2-MeTHF, ${ }^{1}$ Cyrene $^{2}$. Consider catalytic Mitsunobu reaction. ${ }^{18}$

\section{5) Ester Formation}

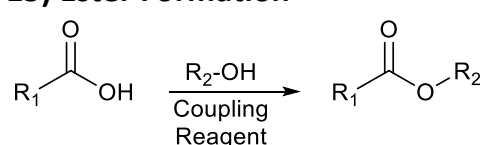

\section{Steglich Esterification}

Potential polar-aprotic alternatives:

EtOAc, 2-MeTHF, ${ }^{1}$ dimethyl carbonate, cyclopentanone ${ }^{29}$, cyclic carbonates, $N$ butylpyrrolidinone (NBP), Cyrene ${ }^{16}, \mathrm{v}$ valerolactone, Cyrene ${ }^{2}$

Fisher Esterification: Usually conducted in the alcohol corresponding to the desired ester chain length.
12) Sulfonamide Formation

$\mathrm{DABSO} / \mathrm{R}_{2}-\mathrm{MgX}$

$\mathrm{R}_{1}-\mathrm{NH}_{2}$

$$
\begin{aligned}
& / \mathrm{NaOCl}
\end{aligned}
$$

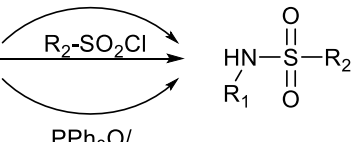

Consider using an alternative to DCM such as EtOAc. ${ }^{19}$ Aqueous and biphasic conditions also possible. ${ }^{20}$

16) Oxidation

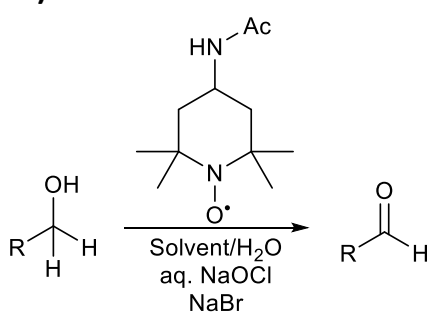

$\mathrm{N}$-Oxy catalysed bleach oxidation of alcohols: Can be conducted in methyl acetate and isopropylacetate instead of DCM. ${ }^{30}$

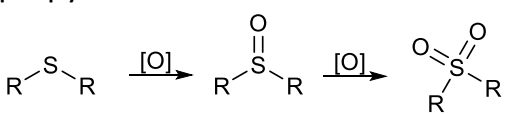

Sulfide oxidation to sulfoxide/sulfone:

Halogen-free biphasic conditions. ${ }^{31}$ 


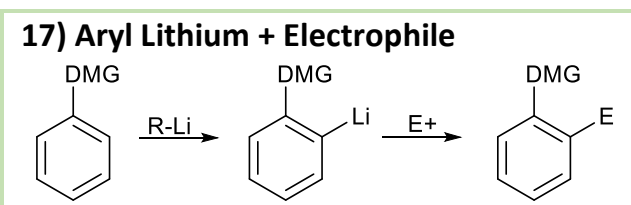

$\mathrm{DMG}=3^{\circ}$ Amide,

O-Carbamate etc.

\section{General advice:}

Try $\mathrm{CPME}^{32}$ or $2-\mathrm{MeTHF}^{33}$ before THF. If you require an alkane solvent then heptane and cyclohexane are both preferable to hexane or petroleum ether. $^{34}$

21) Free-Radical Halogenation - Benzylic Bromination

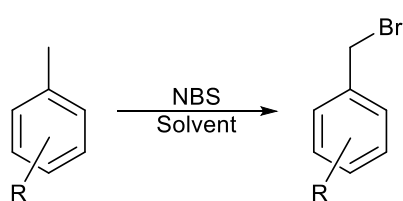

Benzylic Bromination using NBS:

Successfully carried out using

cyclohexane $\mathrm{c}^{45-47}, \mathrm{MeOAc}^{48}$, and iso-

propylacetate ${ }^{49}$ as solvents.

Microwave assisted benzylic bromination in EtOAc and diethyl carbonate have been reported. ${ }^{50}$

Also consider: "on water" benzylic bromination $^{51}$ and solvent-free methods. ${ }^{52}$

\section{8) Wittig Reaction Manifold}

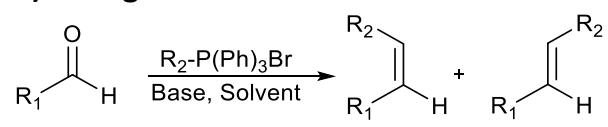

Study of solvent effects in the Wittig

reaction. ${ }^{35}$ Solvent effects are myriad and

complex.

Consider including alcohols $\left(\mathrm{EtOH}^{36}\right.$

$\mathrm{MeOH}^{35}$ ), $\mathrm{EtOAc}^{37}$, and water ${ }^{35}$ in any

solvent screening conducted. Attempt

solvent-free protocols if appropriate. ${ }^{38-39,} 40$

\section{2) Aromatic Halogenation}

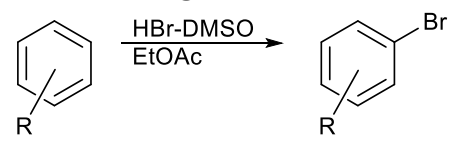

Aromatic bromination can be conducted using $\mathrm{HBr}$ and $\mathrm{H}_{2} \mathrm{O}_{2}$ in methanol, ethanol, water, or under solvent-free conditions. ${ }^{53}$

Bromination using DMSO-HBr or iodination using DMSO- $\mathrm{NH}_{4}$ l have been conducted using EtOAc as solvent. ${ }^{54}$

Heteroarene halogenation using

trihaloisocyanuric acids have been accomplished using EtOH as solvent. ${ }^{55}$
19) Acid Chloride Formation

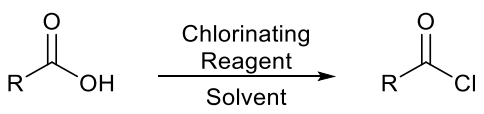

Potential polar-aprotic alternatives: EtOAc, 2-MeTHF ${ }^{41}$, dimethyl carbonate, cyclic carbonates, $N$-butylpyrrolidinone (NBP), $y$-valerolactone, Cyrene ${ }^{2}$.

\section{3) Alcohol}

Halogenation/Deoxyhalogenation -

\section{Appel Reaction}

$(\mathrm{COCl})_{2}$
cat. $\mathrm{P}(\mathrm{Ph})_{3} \mathrm{O}$

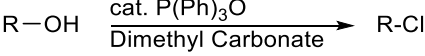

$\mathrm{R}=$ Alkyl, Allyl, Propargyl

\section{Solvent-Reagent Guide:}

More sustainable catalytic Appel bromination and chlorination using

dimethyl carbonate as solvent. ${ }^{56}$

Deoxyfluorination of alcohols can be achieved in a two-step process using DIC and $\mathrm{CuF}_{2}$ in $\mathrm{CPME}$ and $\mathrm{H}_{2} \mathrm{O}$ respectively.
20) Alkene/Alkyne Halogenation

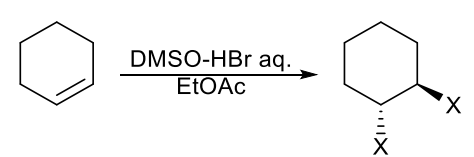

\section{DMSO-based oxidative}

bromination: Conducted in

EtOAc. ${ }^{42}$ Attempt alternative methodologies such as this before considering $\mathrm{Br}_{2}$ based systems.

Oxidative Halogenation review. ${ }^{43}$

Electrophilic iodination methods. ${ }^{44}$

\section{4) Ketone $\alpha$-Halogenation and}

Halogen Addition Reactions

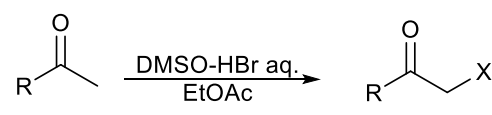

\section{Oxidative bromination (DMSO-}

$\mathrm{HBr}$ ): Conducted in EtOAc. ${ }^{42}$

Attempt methodologies such as

this before considering $\mathrm{Br}_{2}$ based systems.

$\mathrm{Cu}(\mathrm{II}) \mathrm{Br}$ : Reactions reported in 2$\mathrm{EtOH}^{58}, \mathrm{EtOAC}^{59}$ and 2-MeTHF ${ }^{60}$.

$\mathrm{N}$-Halo Succinimide: Screen

alternative solvents such as EtOAc. ${ }^{61}$

$\mathrm{Br}_{2}$ : Screen alcohols (such as $\mathrm{MeOH}^{62}$ ), and EtOAc ${ }^{63}$ as

alternatives to chloroform in $\mathrm{Br}_{2} \alpha$ bromination. 
25) Nucleophilic Fluorination

$\widehat{R}_{L G} \underset{\text { Solvent }}{\mathrm{F}-} \mathrm{R} \widehat{\mathrm{F}}_{\mathrm{F}}$

DAST and Deoxofluor Mediated

Fluorination: Reported use of EtOAc in

both DAST ${ }^{64}$ and Deoxofluor ${ }^{65}$ reactions.

TBAF: TBAF-THF solutions are

commonplace, though THF is suspected of causing cancer (H351). Switching to 2-

MeTHF may be preferable.

See main manuscript for selected

fluorination reviews. ${ }^{66-71}$

Key:

1) Amide Bond Formation

2) $S_{N A r}$

3) Boc Protection

4) Boc Deprotection

5) Ester Hydrolysis

6) Cross- Coupling

7) C-H Activation

8) Amine-Electrophile Reactions

9) Reductive Amination

10) Debenzylation
26) Electrophilic Fluorination

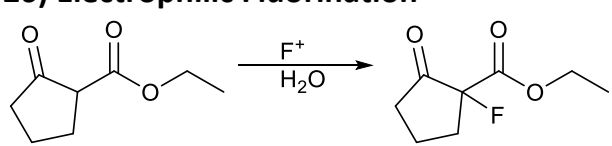

Electrophilic Fluorination has been

conducted in water using Selectfluor ${ }^{68}$, $\mathrm{NFSi}^{72}$, and F-TEDA-BF ${ }_{4}{ }^{73}$

\section{7) Aryl Fluorination}

Metal catalysed aryl fluorination solvents are varied and include cyclohexane, TBME, toluene and 2-MeTHF. ${ }^{74-75}$
11) Heteroatom Alkylation
12) Sulfonamide Formation
13) $\mathrm{NO}_{\mathrm{x}}$ and Nitrile Reduction
14) Diazotization
15) Ester Formation
16) Oxidation
17) Aryl Lithium + Electrophile
18) Wittig Reaction Manifold
19) Acid Chloride Formation
20) Alkene/Alkyne Halogenation
21) Free-Radical Halogenation Benzylic Bromination
28) Biphasic/Schotten-

Baumann/Micellar Conditions

Use a non-chlorinated organic

component that is immiscible with

water such as EtOAc, iso-propyl

acetate $^{76}$, toluene ${ }^{77}$ or 2-MeTHF ${ }^{33,78}$

Consider TPGS-750M surfactant systems

for cross-coupling reactions. ${ }^{7-8}$
28) Silica Gel Chromatography

DCM-MeOH chromatography can be entirely replaced with binary

EtOAc-Heptane or ternary

(EtOAc:EtOH 3:1)-Heptane. ${ }^{79}$

See guide by Taygerly et al. for chlorinated solvent free

chromatography. ${ }^{79}$
22) Aromatic Halogenation

23) Alcohol

Halogenation/Deoxyhalogenat

ion - Appel Reaction

24) Ketone $\alpha$-Halogenation and Halogen Addition Reactions

25) Nucleophilic Fluorination

26) Electrophilic Fluorination

27) Aryl Fluorination

28) Biphasic/Schotten-Baumann Conditions

29) Silica Gel Chromatography
See main review text for in-depth discussions and further examples.

Disclaimer: Advice provided in this guide in no way represents a risk assessment or chemical compatibility assessment. The author's take no responsibility for practitioners safety or reaction outcomes. Adequate risk assessment and safety precautions must be taken when planning any chemical process or transformation and are the responsibility of the practitioner.

Reactions are organised in approximate order of prevalence of use according to Brown et al. ${ }^{80}$ 


\section{References:}

1. MacMillan, D. S.; Murray, J.; Sneddon, H. F.; Jamieson, C.; Watson, A. J. B., Evaluation of alternative solvents in common amide coupling reactions: replacement of dichloromethane and N,N-dimethylformamide. Green Chem. 2013, 15, 596-600.

2. Wilson, K. L.; Murray, J.; Jamieson, C.; Watson, A. J. B., Cyrene as a bio-based solvent for HATU mediated amide coupling. Org. Biomol. Chem. 2018, 16, 2851-2854.

3. Walsh, K.; Sneddon, H. F.; Moody, C. J., Amination of Heteroaryl Chlorides: Palladium Catalysis or SNAr in Green Solvents? ChemSusChem 2013, 6, 1455-1460.

4. Campestre, C.; Keglevich, G.; Kóti, J.; Scotti, L.; Gasbarri, C.; Angelini, G., Microwave-assisted simple synthesis of 2-

anilinopyrimidines by the reaction of 2-chloro-4,6-dimethylpyrimidine with aniline derivatives. RSC Adv. $2020,10,12249-12254$.

5. Rawal, V. H.; Cava, M. P., Thermolytic removal of t-butyloxycarbonyl (BOC) protecting group on indoles and pyrroles. Tetrahedron Lett. 1985, 26, 6141-6142.

6. Hardouin, C.; Lemaitre, S., Safety Case Study. Intrinsic Instability of Concentrated Solutions of Alcoholic Hydrogen Chloride: Potential Hazards Associated with Methanol. Org. Process Res. Dev. 2020, 24, 867-871.

7. Krasovskiy, A.; Duplais, C.; Lipshutz, B. H., Zn-Mediated, Pd-Catalyzed Cross-Couplings in Water at Room Temperature Without Prior Formation of Organozinc Reagents. J. Am. Chem. Soc. 2009, 131, 15592-15593.

8. Wagner, P.; Bollenbach, M.; Doebelin, C.; Bihel, F.; Bourguignon, J.-J.; Salomé, C.; Schmitt, M., t-BuXPhos: a highly efficient ligand for Buchwald-Hartwig coupling in water. Green Chem. 2014, 16, 4170-4178.

9. Skowerski, K.; Białecki, J.; Tracz, A.; Olszewski, T. K., An attempt to provide an environmentally friendly solvent selection guide for olefin metathesis. Green Chem. 2014, 16, 1125-1130.

10. Parker, H. L.; Sherwood, J.; Hunt, A. J.; Clark, J. H., Cyclic Carbonates as Green Alternative Solvents for the Heck Reaction. ACS Sustainable Chemistry \& Engineering 2014, 2, 1739-1742.

11. Yousaf, M.; Zahoor, A. F.; Akhtar, R.; Ahmad, M.; Naheed, S., Development of green methodologies for Heck, Chan-Lam, Stille and Suzuki cross-coupling reactions. Mol. Divers. 2019.

12. Strappaveccia, G.; Ismalaj, E.; Petrucci, C.; Lanari, D.; Marrocchi, A.; Drees, M.; Facchetti, A.; Vaccaro, L., A biomass-derived safe medium to replace toxic dipolar solvents and access cleaner Heck coupling reactions. Green Chem. $2015,17,365-372$.

13. Sherwood, J.; Clark, J. H.; Fairlamb, I. J. S.; Slattery, J. M., Solvent effects in palladium catalysed cross-coupling reactions. Green Chem. 2019, 21, 2164-2213.

14. Sherwood, J., European Restrictions on 1,2-Dichloroethane: C-H Activation Research and Development Should Be Liberated and not Limited. Angew. Chem. Int. Ed. 2018, 57, 14286-14290.

15. Yu, C. ; Sanjosé-Orduna, J.; Patureau, F. W.; Pérez-Temprano, M. H., Emerging unconventional organic solvents for C-H bond and related functionalization reactions. Chem. Soc. Rev. 2020, 49, 1643-1652.

16. Sherwood, J.; De bruyn, M.; Constantinou, A.; Moity, L.; McElroy, C. R.; Farmer, T. J.; Duncan, T.; Raverty, W.; Hunt, A. J.;

Clark, J. H., Dihydrolevoglucosenone (Cyrene) as a bio-based alternative for dipolar aprotic solvents. Chem. Commun. 2014, 50, 96509652. 
17. McGonagle, F. I.; MacMillan, D. S.; Murray, J.; Sneddon, H. F.; Jamieson, C.; Watson, A. J. B., Development of a solvent selection guide for aldehyde-based direct reductive amination processes. Green Chem. 2013, 15, 1159-1165.

18. Beddoe, R. H.; Andrews, K. G.; Magné, V.; Cuthbertson, J. D.; Saska, J.; Shannon-Little, A. L.; Shanahan, S. E.; Sneddon, H. F.; Denton, R. M., Redox-neutral organocatalytic Mitsunobu reactions. Science 2019, 365, 910-914.

19. Muraglia, M.; Franchini, C.; Corbo, F.; Scilimati, A.; Tortorella, V.; Sinicropi, M. S.; De Luca, A.; De Bellis, M.; Camerino, D. C., Synthesis of beta-proline like derivatives and their evaluation as sodium channel blockers. J. Heterocycl. Chem. 2007, 44, 1099-1103.

20. Deng, X.; Mani, N. S., A facile, environmentally benign sulfonamide synthesis in water. Green Chem. $2006,8,835-838$.

21. Nitro Reduction Reaxys Search as of 22/04/2020.

22. Whritenour, D. C.; Brenek, S. J.; Tom, N. J., An Improved Procedure for the Reduction of 2,4-Dinitrobenzaldehyde to 2,4-

Diaminobenzaldehyde with Iron and Acetic Acid under Dose-Controlled Conditions. Org. Process Res. Dev. $2001,5,539-541$.

23. Theunissen, C.; Métayer, B.; Henry, N.; Compain, G.; Marrot, J.; Martin-Mingot, A.; Thibaudeau, S.; Evano, G., Keteniminium

Ion-Initiated Cascade Cationic Polycyclization. J. Am. Chem. Soc. 2014, 136, 12528-12531.

24. Sandmeyer-like Reaxys Search as of 23/04/2020.

25. Masllorens, J.; Moreno-Mañas, M.; Pla-Quintana, A.; Roglans, A., First Heck Reaction with Arenediazonium Cations with Recovery of Pd-Triolefinic Macrocyclic Catalyst. Org. Lett. 2003, 5, 1559-1561.

26. Taylor, J. G.; Correia, C. R. D., Stereoselective Synthesis of Unsymmetrical $\beta$, $\beta$-Diarylacrylates by a Heck-Matsuda Reaction:

Versatile Building Blocks for Asymmetric Synthesis of $\beta, \beta$-Diphenylpropanoates, 3-Aryl-indole, and 4-Aryl-3,4-dihydro-quinolin-2-one and Formal Synthesis of (-)-Indatraline. J. Org. Chem. 2011, 76, 857-869.

27. Laudien, J.; Fouquet, E.; Zakri, C.; Felpin, F.-X., A Multi-Task Palladium Catalyst Involved in Heck-Reduction-Cyclization

Sequences for the Preparation of 4-Benzyl-1,2-dihydroisoquinolin-3-ones: An Unusual Homogeneous-Heterogeneous Sustainable Approach. Synlett 2010, 2010, 1539-1543.

28. Perez, R.; Veronese, D.; Coelho, F.; Antunes, O. A. C., Palladium catalyzed Heck reaction of arenediazonium tetrafluoroborate salts with Baylis-Hillman adducts: production of a-benzyl- $\beta$-keto esters. Tetrahedron Lett. 2006, 47, 1325-1328.

29. Jordan, A.; Sneddon, H. F., Development of a solvent-reagent selection guide for the formation of thioesters. Green Chem. 2019, 21, 1900-1906.

30. Janssen, M. H. A.; Chesa Castellana, J. F.; Jackman, H.; Dunn, P. J.; Sheldon, R. A., Towards greener solvents for the bleach oxidation of alcohols catalysed by stable N-oxy radicals. Green Chem. 2011, 13, 905-912.

31. Sato, K.; Hyodo, M.; Aoki, M.; Zheng, X.-Q.; Noyori, R., Oxidation of sulfides to sulfoxides and sulfones with $30 \%$ hydrogen peroxide under organic solvent- and halogen-free conditions. Tetrahedron 2001, 57, 2469-2476.

32. Mallardo, V.; Rizzi, R.; Sassone, F. C.; Mansueto, R.; Perna, F. M.; Salomone, A.; Capriati, V., Regioselective desymmetrization of diaryltetrahydrofurans via directed ortho-lithiation: an unexpected help from green chemistry. Chem. Commun. 2014, 50, 8655-8658.

33. Pace, V.; Hoyos, P.; Castoldi, L.; Domínguez de María, P.; Alcántara, A. R., 2-Methyltetrahydrofuran (2-MeTHF): A BiomassDerived Solvent with Broad Application in Organic Chemistry. ChemSusChem 2012, 5, $1369-1379$.

34. Alder, C. M.; Hayler, J. D.; Henderson, R. K.; Redman, A. M.; Shukla, L.; Shuster, L. E.; Sneddon, H. F., Updating and further expanding GSK's solvent sustainability guide. Green Chem. 2016, 18, 3879-3890. 
35. El-Batta, A.; Jiang, C.; Zhao, W.; Anness, R.; Cooksy, A. L.; Bergdahl, M., Wittig Reactions in Water Media Employing Stabilized Ylides with Aldehydes. Synthesis of a, $\beta$-Unsaturated Esters from Mixing Aldehydes, a-Bromoesters, and Ph3P in Aqueous NaHCO3. J. Org. Chem. 2007, 72, 5244-5259.

36. Chiellini, G.; Rapposelli, S.; Zhu, J.; Massarelli, I.; Saraceno, M.; Bianucci, A. M.; Plum, L. A.; Clagett-Dame, M.; DeLuca, H. F., Synthesis and biological activities of vitamin D-like inhibitors of CYP24 hydroxylase. Steroids 2012, 77, 212-223.

37. Alonso-Fernández, J.; Benaiges, C.; Casas, E.; Alibés, R.; Bayón, P.; Busqué, F.; Álvarez-Larena, Á.; Figueredo, M., Synthetic studies on Stemona alkaloids. Construction of the sessilifoliamides B and C and 1,12-secostenine skeleton. Tetrahedron 2016, 72, 35003524.

38. Morsch, L. A.; Deak, L.; Tiburzi, D.; Schuster, H.; Meyer, B., Green Aqueous Wittig Reaction: Teaching Green Chemistry in Organic Teaching Laboratories. J. Chem. Educ. 2014, 91, 611-614.

39. Leung, S. H.; Angel, S. A., Solvent-Free Wittig Reaction: A Green Organic Chemistry Laboratory Experiment. J. Chem. Educ.

2004, 81, 1492

40. Robertson, F. J., A Highly Versatile One-Pot Aqueous Wittig Reaction. World J. Chem. Educ. 2016, 4, 101-106.

41. Hanson, R. L.; Johnston, R. M.; Goldberg, S. L.; Parker, W. L.; Goswami, A., Enzymatic Preparation of an R-Amino Acid

Intermediate for a $Y$-Secretase Inhibitor. Org. Process Res. Dev. 2013, 17, 693-700.

42. Song, S.; Li, X.; Sun, X.; Yuan, Y.; Jiao, N., Efficient bromination of olefins, alkynes, and ketones with dimethyl sulfoxide and hydrobromic acid. Green Chem. 2015, 17, 3285-3289.

43. Podgoršek, A.; Zupan, M.; Iskra, J., Oxidative Halogenation with "Green" Oxidants: Oxygen and Hydrogen Peroxide. Angew.

Chem. Int. Ed. 2009, 48, 8424-8450.

44. Stavber, S.; Jereb, M.; Zupan, M., Electrophilic Iodination of Organic Compounds Using Elemental Iodine or Iodides. Synthesis

2008, 2008, 1487-1513.

45. Dehimat, Z. I. ; Paşahan, A.; Tebbani, D.; Yaşar, S.; Özdemir, İ., Synthesis of sterically hindered N-benzyladamantyl substituted benzimidazol-2-ylidene palladium complexes and investigation of their catalytic activity in aqueous medium. Tetrahedron $\mathbf{2 0 1 7}, 73$ 5940-5945.

46. Krishnamurthy, M.; Schirle, N. T.; Beal, P. A., Screening helix-threading peptides for RNA binding using a thiazole orange displacement assay. Biorg. Med. Chem. 2008, 16, 8914-8921.

47. Krishnamurthy, M.; Simon, K.; Orendt, A. M.; Beal, P. A., Macrocyclic Helix-Threading Peptides for Targeting RNA. Angew. Chem. Int. Ed. 2007, 46, 7044-7047.

48. Amijs, C. H. M.; van Klink, G. P. M.; van Koten, G., Carbon tetrachloride free benzylic brominations of methyl aryl halides. Green Chem. 2003, 5, 470-474.

49. Mestres, R.; Palenzuela, J., High atomic yield bromine-less benzylic bromination. Green Chem. 2002, $4,314-316$.

50. Pingali, S. R. K.; Upadhyay, S. K.; Jursic, B. S., Microwave-assisted benzyl mono- and dibromination in diethyl carbonate as environmentally friendly alternative to radical bromination in carbon tetrachloride. Green Chem. 2011, 13, 928-933.

51. Podgoršek, A.; Stavber, S.; Zupan, M.; Iskra, J., Visible light induced 'on water' benzylic bromination with N-bromosuccinimide. Tetrahedron Lett. 2006, 47, 1097-1099.

52. Jereb, M.; Zupan, M.; Stavber, S., Visible-Light-Promoted Wohl-Ziegler Functionalization of Organic Molecules with NBromosuccinimide under Solvent-Free Reaction Conditions. Helv. Chim. Acta 2009, 92, 555-566. 
53. Kajorinne, J. K.; Steers, J. C. M.; Merchant, M. E.; MacKinnon, C. D., Green halogenation reactions for (hetero)aromatic ring systems in alcohol, water, or no solvent. Can. J. Chem. 2018, 96, 1087-1091.

54. Song, S.; Sun, X.; Li, X.; Yuan, Y.; Jiao, N., Efficient and Practical Oxidative Bromination and Iodination of Arenes and Heteroarenes with DMSO and Hydrogen Halide: A Mild Protocol for Late-Stage Functionalization. Org. Lett. 2015, 17, $2886-2889$.

55. Neto, J. S. S.; Balaguez, R. A.; Franco, M. S.; de Sá Machado, V. C.; Saba, S.; Rafique, J.; Galetto, F. Z.; Braga, A. L., Trihaloisocyanuric acids in ethanol: an eco-friendly system for the regioselective halogenation of imidazo-heteroarenes. Green Chem. 2020, 22, 3410-3415.

56. Jordan, A.; Denton, R. M.; Sneddon, H. F., Development of a More Sustainable Appel Reaction. ACS Sustainable Chemistry \& Engineering 2020, 8, 2300-2309.

57. Sood, D. E.; Champion, S.; Dawson, D. M.; Chabbra, S.; Bode, B. E.; Sutherland, A.; Watson, A. J. B., Deoxyfluorination with CuF2: Enabled by Using a Lewis Base Activating Group. Angew. Chem. Int. Ed. 2020, 59, 8460-8463.

58. Shi, L.; Hu, A.; Xu, J.; Jiang, Y., Design, Synthesis and Biological Evaluation of 2-(2-Aryl-morpholino-4-yl)ethyl Esters of Indomethacin as Potential Cyclooxygenase-2 (COX-2) Inhibitors. Chin. J. Chem . 2012, 30, 1339-1344.

59. Sahin, Z.; Ertas, M.; Berk, B.; Biltekin, S. N.; Yurttas, L.; Demirayak, S., Studies on non-steroidal inhibitors of aromatase enzyme; 4-(aryl/heteroaryl)-2-(pyrimidin-2-yl)thiazole derivatives. Biorg. Med. Chem. 2018, 26, 1986-1995.

60. Sanivarapu, S.; Vaddiraju, N.; Velide, L., Synthesis and anti-inflammatory activity of 1,2-3-substituted 2a1,4,5-

triazacyclopenta[cd]indene derivatives. Med. Chem. Res. 2019, 28, 1461-1470.

61. Meshram, H. M.; Reddy, P. N.; Sadashiv, K.; Yadav, J. S., Amberlyst-15®-promoted efficient 2-halogenation of 1,3-keto-esters and cyclic ketones using N-halosuccinimides. Tetrahedron Lett. 2005, 46, 623-626.

62. Szántay, C.; Kardos-Balogh, Z.; Moldvai, I.; Szántay, C.; Temesvári-Major, E.; Blaskó, G., A practical enantioselective synthesis of epibatidine. Tetrahedron 1996, 52, 11053-11062.

63. Narayana, B.; Vijaya Raj, K. K.; Ashalatha, B. V.; Kumari, N. S., Synthesis of Some New 4-(2-Chloropyridin-4-yl)-N-Aryl-1,3Thiazol-2-Amine Derivatives as Possible Antifungal and Antibacterial Agents. Phosphorus Sulfur Silicon Relat. Elem. 2007, 182, 7-14. 64. Dinnell, K.; Elliott, J. M.; Hollingworth, G. J.; Ridgill, M. P.; Shaw, D. E. 2-aryl indole derivatives and their use as therapeutic agents. U.S. Patent 2001/0039286A1, Nov. 8, 2001

65. Hewawasam, P.; Kadow, J. F.; Lopez, O. D.; Meanwell, N. A.; Tu, Y.; Wang, A. X.; Xu, N.; Gupta, S. V. S. A. K.; Srinivasu, P.; Kumar, I. G.; Kumar, P. S.; Belema, M.; Fridell, R. A.; Gao, M.; Lemm, J. A.; O'boyle II, D. R.; Sun, J.-h.; Wang, C.; Wang, Y.-k. Hepatitis C Virus Inhibitors. U.S. Patent 2013/0183269A1, July 18, 2013.

66. Szpera, R.; Moseley, D. F. J.; Smith, L. B.; Sterling, A. J.; Gouverneur, V., The Fluorination of C-H Bonds: Developments and Perspectives. Angew. Chem. Int. Ed. 2019, 58, 14824-14848.

67. Cheng, Q.; Ritter, T., New Directions in C-H Fluorination. Trends Chem. 2019, 1, 461-470.

68. Yang, L.; Dong, T.; Revankar, H. M.; Zhang, C.-P., Recent progress on fluorination in aqueous media. Green Chem. 2017, 19, 3951-3992.

69. Yerien, D. E.; Bonesi, S.; Postigo, A., Fluorination methods in drug discovery. Org. Biomol. Chem. 2016, 14, 8398-8427.

70. Champagne, P. A.; Desroches, J.; Hamel, J.-D.; Vandamme, M.; Paquin, J.-F., Monofluorination of Organic Compounds: 10 Years of Innovation. Chem. Rev. 2015, 115, 9073-9174. 

480.

Adams, J. P.; Alder, C. M.; Andrews, I.; Bullion, A. M.; Campbell-Crawford, M.; Darcy, M. G.; Hayler, J. D.; Henderson, R. K.; Oare, C. A.; Pendrak, I.; Redman, A. M.; Shuster, L. E.; Sneddon, H. F.; Walker, M. D., Development of GSK's reagent guides embedding sustainability into reagent selection. Green Chem. 2013, 15, 1542-1549.

73. Stavber, G.; Stavber, S., Towards Greener Fluorine Organic Chemistry: Direct Electrophilic Fluorination of Carbonyl Compounds in Water and Under Solvent-Free Reaction Conditions. Adv. Synth. Catal. 2010, 352, 2838-2846.

74. Lee, H. G.; Milner, P. J.; Buchwald, S. L., Pd-Catalyzed Nucleophilic Fluorination of Aryl Bromides. J. Am. Chem. Soc. 2014, 136, 3792-3795.

75. Sather, A. C.; Buchwald, S. L., The Evolution of $\mathrm{Pd}(0) / \mathrm{Pd}(\mathrm{II})$-Catalyzed Aromatic Fluorination. Acc. Chem. Res. 2016, 49, 21462157.

76. White, T. D.; Berglund, K. D.; Groh, J. M.; Johnson, M. D.; Miller, R. D.; Yates, M. H., Development of a Continuous SchottenBaumann Route to an Acyl Sulfonamide. Org. Process Res. Dev. 2012, 16, 939-957.

77. Jordan, A.; Haiß, A.; Spulak, M.; Karpichev, Y.; Kümmerer, K.; Gathergood, N., Synthesis of a series of amino acid derived ionic liquids and tertiary amines: green chemistry metrics including microbial toxicity and preliminary biodegradation data analysis. Green Chem. 2016, 18, 4374-4392.

78. Brown Ripin, D. H.; Vetelino, M., 2-Methyltetrahydrofuran as an Alternative to Dichloromethane in 2-Phase Reactions. Synlett 2003, 2003, 2353-2353.

79. Taygerly, J. P.; Miller, L. M.; Yee, A.; Peterson, E. A., A convenient guide to help select replacement solvents for dichloromethane in chromatography. Green Chem. 2012, 14, 3020-3025.

80. Brown, D. G.; Boström, J., Analysis of Past and Present Synthetic Methodologies on Medicinal Chemistry: Where Have All the New Reactions Gone? J. Med. Chem. 2016, 59, 4443-4458. 